\title{
Light field and crosshair quality assurance test using a simple lens system
}

\author{
Scott W. Hadley and Kwok Lam \\ Department of Radiation Oncology, University of Michigan, Ann Arbor, Michigan 48109-0010
}

(Received 7 June 2005; revised 23 January 2006; accepted for publication 24 January 2006; published 17 March 2006)

\begin{abstract}
We describe here a simple lens system to test the positioning of the field light source and mylar crosshair for radiation therapy linear accelerators. Ideally the light source for the field light and the crosshair should be centered on the axis of rotation of the collimator. The traditional method for testing this coincidence uses the shadow of the crosshair caused by the field light source. The shadow of the crosshair is dependent on the position of both the field light source and mylar crosshair. Geometrically it is possible for the field light source and the mylar crosshair to be off the axis of rotation of the collimator and still cause the shadow of the crosshair to be on the axis of rotation at some distance. Using a lens system the motion of the field light source and crosshair can be observed in sharp focus independently of one another as the collimator is rotated. (C) 2006 American Association of Physicists in Medicine. [DOI: 10.1118/1.2174130]
\end{abstract}

\section{INTRODUCTION}

Radiation therapy linear accelerators use a light source to project a light field on the patient representing the radiation beam. Additionally there is a mylar crosshair to project a shadow of orthogonal axes whose intersection is the central axis of the radiation beam. For the Varian linear accelerator, the light source and the mylar crosshair are part of the collimator and rotate with it. Ideally the axis of rotation of the collimator would coincide with the central axis of the beam. If the light source and mylar were centered on the axis of rotation of the collimator, then under any rotation of the collimator the light field would retain the correct projection. The light source also projects the position of the jaws and multileaf collimator (MLC). If the light source is not in the correct position, it can shift the position of the light field formed by jaws and MLCs and misrepresent where the radiation beam is targeted. AAPM Report No. 46 from Task Group 40 recommends testing the radiation and light field agreement on a monthly basis with a tolerance of $2 \mathrm{~mm}$ or $1 \%$ on any side. ${ }^{1}$

Two methods can be used to verify the position of the field light source and mylar crosshair. One is to adjust the two until the shadow of the crosshair does not wander when viewed at two different distances from the light source. This method can be difficult because any wander in the shadow of the crosshair could be a combination of an error in both the crosshair and the light source.

The other is to place an object, like a paper clip, external to the collimator to cast a shadow on the floor. The field light source position is adjusted until the shadow does not wander when the collimator is rotated. One problem with this is that the magnification of the shadow of the paperclip can be large and the effective size of the light source produces a crosshair shadow with blurry edges. This reduces one's ability to see the wander and adjust the light source.

We present here a simple method to see the wander of the individual components of the light field using a convex lens. The lens is used to focus a sharp image of the light source or crosshair onto a screen and observe any wander as the collimator is rotated. At the same time the lens can magnify the images of each and make any error in adjustment more noticeable. This simple test could be included in linear accelerator commissioning or annual quality assurance tests.

\section{METHODS AND MATERIALS}

Suitable commonly available convex lenses for each test were used to focus the image of the light source or crosshair onto a screen. The focal length of the lens for the light source test can be chosen so that the magnification of the light source is at least as large as the magnification of the upper jaws to the isocenter. For the crosshair, the focal length can be chosen so that the magnification of the crosshair is at least as large as that of the projection of the crosshair at the isocenter. The screen could be as simple as a sheet of graph paper attached to a hard surface. The lens was placed close to the collimator approximately on the central axis with the screen farther away. One adjusts the position of lens and screen to magnify the image on the screen and bring it into focus. Figure 1 shows the typical setup for the field light source and crosshair.

Using the thin lens approximation, $1 / f=1 / o+1 /(F+f)$, the magnification is $M=F / f$ where $f$ is the focal length of the lens, $F$ is the distance from the focal spot to the screen, and $o$ is the distance from the object to the lens. Any wander in the light source or crosshair will be magnified by that amount.

By rotating the collimator one can observe any wander in either the light source or mylar crosshair independent of one another. An estimate in the radius of the circle traced out can be determined by using graph paper as the screen. Adjustments can then be done to zero out any wander.

There are some practical considerations to keep in mind when choosing lenses for this method. Ideally the magnification factor would be large so that small deviations in the positions would be more apparent. One could use the wall of the treatment room for the position of the screen. This limits the distance from the object to the screen. In order to have a real image that also magnifies the object, the distance from the object to the lens needs to be between $f$ and $2 f$. The 


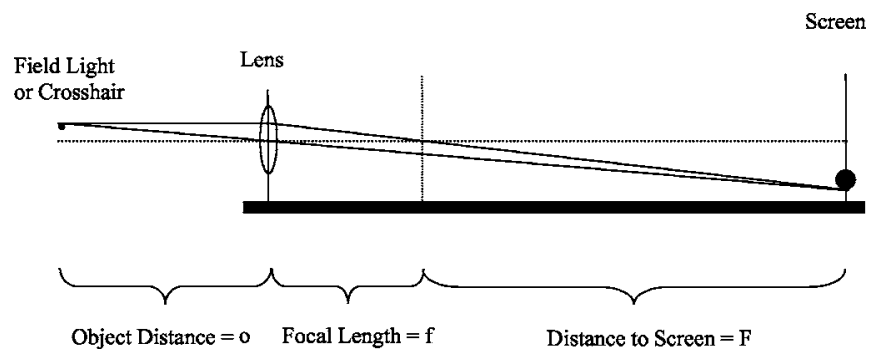

FIG. 1. Schematic diagram of the setup to magnify and focus individual components of the collimator field light and crosshair. A lens is placed outside the collimator and focuses the field light source onto a screen some distance away.

distance from the light source to the lens cannot be less than the distance from the light source to the mylar. That sets the object distance, $o$, of the light source to greater than or equal to $58 \mathrm{~cm}$ on a Varian 2100 accelerator. If the lens is set at the mylar window, then the focal length of the lens needs to be between 58 and $28 \mathrm{~cm}$. If, for example, the far wall at $(f$ $+F)=400 \mathrm{~cm}$ was to be used as the screen, the focal length should be $51 \mathrm{~cm}$. We rotate the gantry to a lateral position and use the treatment couch rotated $90 \mathrm{deg}$ as a bench to support the lens and the screen with $(f+F) \approx 200 \mathrm{~cm}$. Thus the focal length of the lens for the light field was chosen to be $45 \mathrm{~cm}$, which results in a magnification factor of 3.4 . Using a similar setup for the mylar crosshair, the focal length of the lens needs to be less than $50 \mathrm{~cm}$. If one wants to keep the magnification factor the same as the field light, then the focal length should be $35 \mathrm{~cm}$.

This system was tested on a linear accelerator known to have a disagreement between the light and radiation field approaching the $2 \mathrm{~mm}$ tolerance recommended in AAPM Report No. 46 for monthly quality assurance. The placement of the crosshair was confirmed using the standard method and the jaws were recalibrated. Varian accelerators use the shadow of the jaw to measure the position during jaw calibration. If the field light and crosshair are out of alignment with the collimator rotation it can cause the light and radiation fields to disagree even after jaw calibration. The difference between the light field and radiation persisted after calibration and thus the position of the field light and the collimator axis of rotation were suspected as the problem.

The lens system was used to adjust the position of the field light. A $45.0 \mathrm{~cm}$ focal length convex lens was placed close to the mylar crosshair of the machine with the gantry and the couch at $270 \mathrm{deg}$ (Varian IEC scale). This positions the lens at about $65 \mathrm{~cm}$ from the virtual position of the light source. Millimeter graph paper served as the screen and was placed $215 \mathrm{~cm}$ from the lens resulting in $F=170$. The magnification factor was 3.78. This setup focused the light source onto the graph paper to aid in the estimation of the wander. The collimator was rotated $180 \mathrm{deg}$ and the image of the field light was observed to determine what direction to adjust the field light to zero out any wander. Adjustments were made and then the process repeated until the image of the field light did not wander.
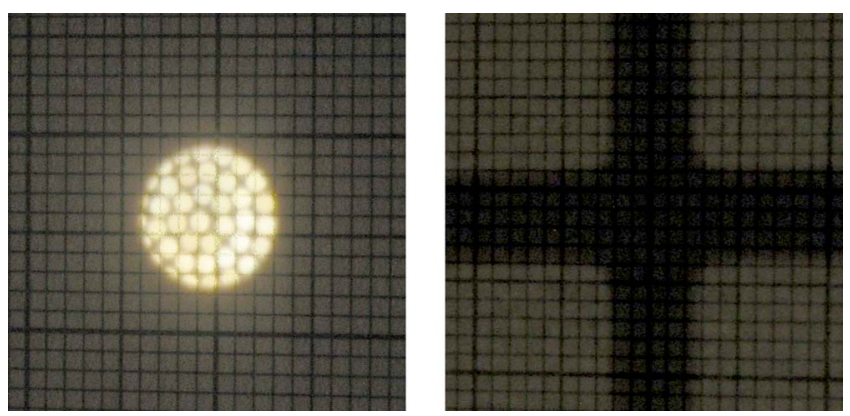

FIG. 2. Examples of field light and crosshair focused onto a millimeter graph paper screen. The image on the left is a field light of a Varian 6/100. The individual fiber optic bundles that make up the light source can be seen on the screen. The image on the right is the crosshair focused on the screen (the field light and crosshair use lenses with different focal lengths). As the collimator rotates these two objects rotate on the screen and wander in their position can be easily seen.

The conventional method was used to place the crosshair on the axis of rotation of the collimator. The jaws were then recalibrated and the light versus radiation field agreement measured again. Our lens system to check the crosshair deviation uses a $20 \mathrm{~cm}$ focal length lens placed $21 \mathrm{~cm}$ from the mylar. The screen sits in the same position as it does for the light source which sets $F=174 \mathrm{~cm}$ and the magnification factor to 8.7 .

To validate this method we used a digital camera with a macro zoom lens to acquire pictures of the light source and crosshair as the collimator rotated. The screen used was a piece of semitransparent tape that held the scale of an eye loupe to a clear filter. This was placed at the same distance as the millimeter graph paper. The scale was used to calibrate the absolute displacement of objects on the screen. Image processing was used to estimate the position of the light source or crosshair as a function of collimator rotation. The

Motion of Light Source and Crosshair Before and After Adjustm ent

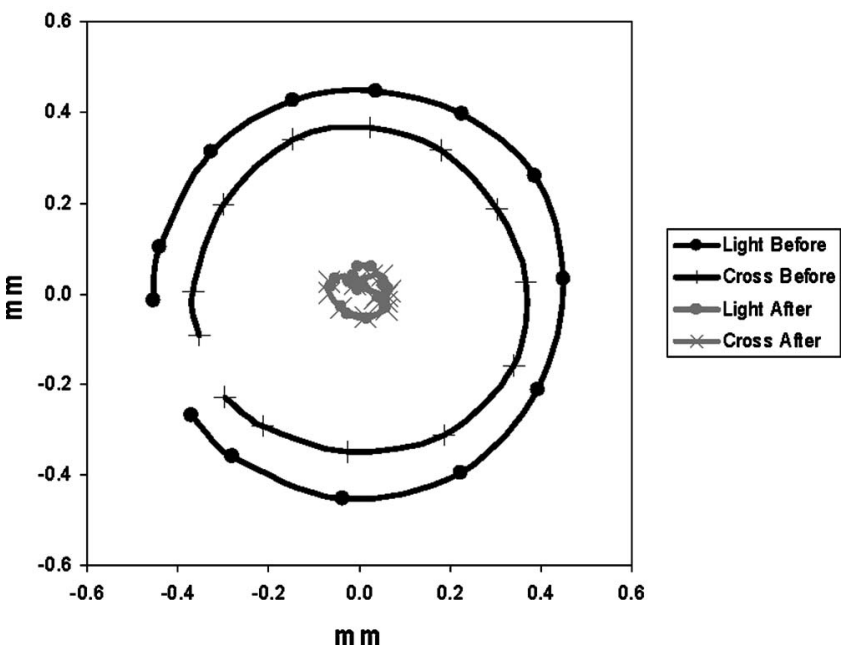

FIG. 3. Graph of the actual motion of the field light and crosshair, before and after adjustment, as the collimator is rotated. 
radius of the circle was estimated by fitting a circle to data. The circle was then demagnified to determine the motion of the light field and crosshair in millimeters.

\section{RESULTS}

Using the lens system a magnified image of the field light was brought into sharp focus. This made observation of any drift in its position easy to determine and correct. Figure 2 shows examples of the field light source and the mylar crosshair focused onto graph paper. The magnification of the light source was 3.8 and the crosshair was 8.7 in these images. The gridline on the graph paper was used to aid in the measurement and adjustment of the light source positioning when the collimator was rotated.

Figure 3 shows a graph of the position of the field light source and mylar crosshair before and after adjustment. This position was measured at the graph paper and demagnified to represent the actual motion of each.

Before adjustment the field light traced out a circle with a diameter of $0.9 \mathrm{~mm}$ and the mylar crosshair $0.7 \mathrm{~mm}$. The light and radiation fields disagreed by as much as $1.5 \mathrm{~mm}$ before adjustment. Using the conventional method the shadow of the wire on the floor had a width of approximately $5 \mathrm{~mm}$ and deviated $2 \mathrm{~mm}$ when the collimator was rotated.
After adjustment it was not possible to estimate a circular path but the collection of points was contained in a space $0.2 \mathrm{~mm}$ by $0.2 \mathrm{~mm}$ for both the field light and the crosshair. The light and radiation field disagreement was too small to measure reliably. Using the shadow of the wire the movement was so small it was difficult to measure any deviation after adjustment.

\section{CONCLUSION}

The conventional method makes observation of the wander of field light source difficult because the wire projects to a large blurry object with a width larger than the deviation to be observed. Using the lens system facilitated the measurement and correction of the placement of the field light source. The main effect was to bring the light source and crosshair individually into sharp focus with sufficient magnification. This makes observation of any wander easy to see and correct. Using the lens system removes the ambiguity of the adjustment and gives the user a quantifiable measurement of the wander of the field light.

${ }^{1}$ G. J. Kutcher, L. Coia, M. Gillin, W. F. Hanson, S. Leibel, R. J. Morton, J. R. Palta, J. A. Purdy, L. E. Reinstein, G. K. Svensson, M. Weller, and L. Wingfield, "Comprehensive QA for radiation oncology: Report of AAPM Radiation Therapy.” Med. Phys. 21(4), 581-618 (1994). 